\title{
Spectroscopy of neutron rich nuclei using cold neutron induced fission of actinide targets at the ILL: the EXILL campaign
}

\author{
G. de France ${ }^{\text {1a }}$, A. Blanc ${ }^{2}$, F. Drouet ${ }^{3}$, M. Jentschel ${ }^{2}$, U. Köster ${ }^{2}$, P. Mutti ${ }^{2}$, J.M. Régis ${ }^{4}$, G. \\ Simpson $^{3}$, T. Soldner ${ }^{2}$, O. Stezowski ${ }^{5}$, C.A. Ur ${ }^{6}$, W. Urban ${ }^{2,7}$, and A. Vancrayenest ${ }^{3}$ \\ ${ }^{1}$ Grand Accélérateur National d'Ions Lourds (GANIL), CEA/DSM-CNRS/IN2P3, Bd Henri \\ Becquerel, BP55027, F-14076 Caen Cedex 5, France \\ ${ }^{2}$ Institut Laue-Langevin, BP 156, 6, rue Jules Horowitz, 38042 Grenoble Cedex 9, France \\ ${ }^{3}$ LPSC, Université Joseph Fourier Grenoble 1, CNRS/IN2P3, Institut National Polytechnique de \\ Grenoble, F-38026 Grenoble Cedex, France \\ ${ }^{4}$ Institut für Kernphysik der Universität zu Köln, Zülpicher Str. 77, 50937 Köln, Germany \\ ${ }^{5}$ Institut de Physique Nucléaire de Lyon CNRS/IN2P3, Université Claude Bernard, 4 rue Enrico \\ Fermi, F-69622 Villeurbanne Cedex, France \\ ${ }^{6}$ INFN Sezione di Padova, I-35131 Padova, Italy \\ ${ }^{7}$ Faculty of Physics, University of Warsaw, ul. Hoza 69, 00-681 Warsaw, Poland
}

\begin{abstract}
A combination of germanium detectors has been installed at the PF1B neutron guide of the ILL to perform the prompt spectroscopy of neutron-rich nuclei produced in the neutron-capture induced-fission of ${ }^{235} \mathrm{U}$ and ${ }^{241} \mathrm{Pu}$. In addition $\mathrm{LaBr}_{3}$ detectors from the FATIMA collaboration have been installed in complement with the EXOGAM clovers to measure lifetimes of low-lying excited states. The measured characteristics and online spectra indicate very good performances of the overall setup.
\end{abstract}

\section{Introduction}

Fission is known since long to produce abundantly neutron-rich nuclei. An excellent method for cleanly producing very neutron-rich fragments is to induce fission reactions in actinide targets using slow neutrons from a neutron guide. In this regime, the neutrons bring just enough energy into the system to produce fission, which preserves the neutron-richness of the fragments. The average spin generated in this reaction is 6-7h [1] and states with spins as high as $20 \hbar$ can be observed. Of particular interest in this context is the use of ${ }^{235} \mathrm{U}$ and ${ }^{241} \mathrm{Pu}$ fissile targets which give access to nuclei that are too weakly produced using spontaneous fission sources or another production mean to perform their prompt spectroscopy. In this contribution we will describe the EXILL campaign which took place recently at the research reactor of the Institut Laue Langevin (ILL), Grenoble, to perform such studies. The setup was also used to measure a few radiative neutron capture reactions.

${ }^{\text {a }}$ Corresponding author: defrance@ganil.fr

This is an Open Access article distributed under the terms of the Creative Commons Attribution License 2.0, which permits unrestricted use, distribution, and reproduction in any medium, provided the original work is properly cited. 


\section{Physics goals}

The basic idea of EXILL (EXogam at the ILL) was to install a large number of efficient germanium detectors around a fissile target irradiated by a well collimated cold neutron beam. We used ${ }^{235} \mathrm{U}$ and ${ }^{241} \mathrm{Pu}$ targets giving access to many nuclei where currently nothing or little is known, especially in the regions north-east of ${ }^{78} \mathrm{Ni}$ and beyond ${ }^{132} \mathrm{Sn}$. This is what is shown in figure 1 which compares the mass yield distribution from the thermal neutron induced fission of ${ }^{235} \mathrm{U}$ and ${ }^{241} \mathrm{Pu}$ and the yield from the spontaneous fission of ${ }^{252} \mathrm{Cf}$ and ${ }^{248} \mathrm{Cm}$. The production of nuclei in the mass A 90 region is an order of magnitude larger using a ${ }^{235} \mathrm{U}$ target compared to the spontaneous fission sources. The same is true for nuclei in the mass $\mathrm{A} \sim 125$ region using a ${ }^{241} \mathrm{Pu}$ target. These two mass regions were the focus of the EXILL physics case which is briefly described in the following.

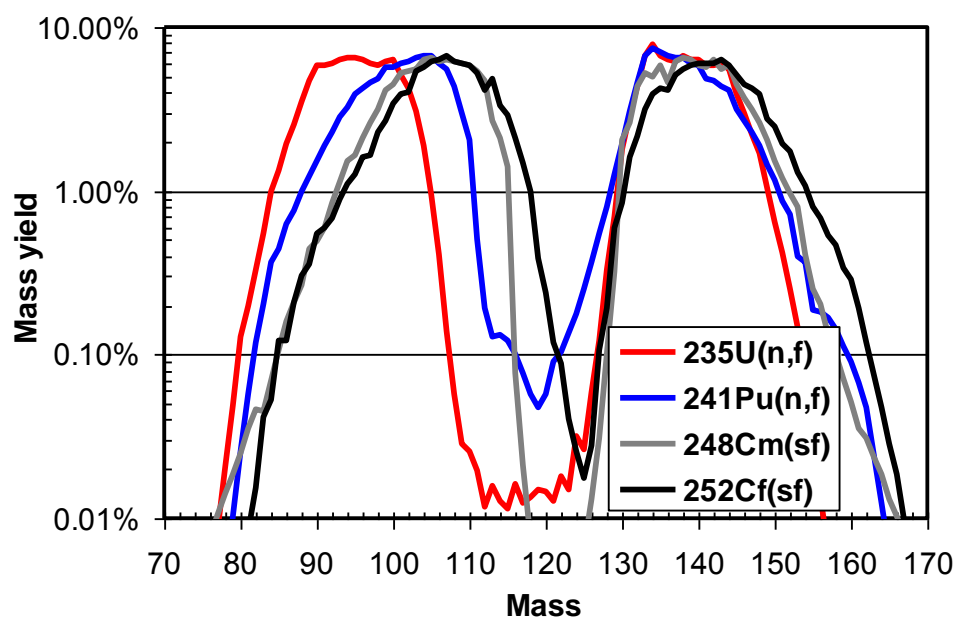

Figure 1. Mass yield distributions measured for the spontaneous fission of ${ }^{252} \mathrm{Cf}$ and ${ }^{248} \mathrm{Cm}$ compared to the thermal neutron induced fission of ${ }^{235} \mathrm{U}$ and ${ }^{241} \mathrm{Pu}$ (data from [2]).

Nuclei with just a few particles or holes outside a double shell closure like ${ }^{132} \mathrm{Sn}$ allow sensitive tests of the interactions used in shell-model (SM) calculations to be performed. One way to address this question, is to obtain spectroscopic informations in these nuclei. Correct predictions of nuclear structure are important as nuclei here lie on, or next to, the astrophysical rapid neutron capture process. Properties, such as neutron binding energies and low-lying decaying states can change the speed and direction of the predicted r-process path.

The evolution of the $\mathrm{N}=50$ gap in exotic nuclei and the question of whether ${ }^{78} \mathrm{Ni}$ is a doubly magic nucleus is highly debated. From the structure of nuclei with $\mathrm{N}=49-50$ close to ${ }^{78} \mathrm{Ni}$, one can see how well SM interactions can reproduce excited states and to what level contributions from core excitations need to be included. In particular, we are missing the single-particle energies and the two body matrix elements for the residual proton-neutron interactions.

The reasons for the rapid change from a spherical to a deformed shape in the A 100 region, with the addition of just a few neutrons, remained a mystery for a long time. In recent years, studies point to the role played by deformation-driving, and resisting, Nilsson orbitals. However, such arguments neglect any role played by the protons in such phenomena and the neutron-proton interaction is thought to be largely responsible for the onset of collectivity when moving away from closed shells. Studies of the intermediate spin states of the $\mathrm{Kr}$ and $\mathrm{Rb}$ nuclei with $\mathrm{N} \sim 59$ will help to clarify the role played by the protons. 

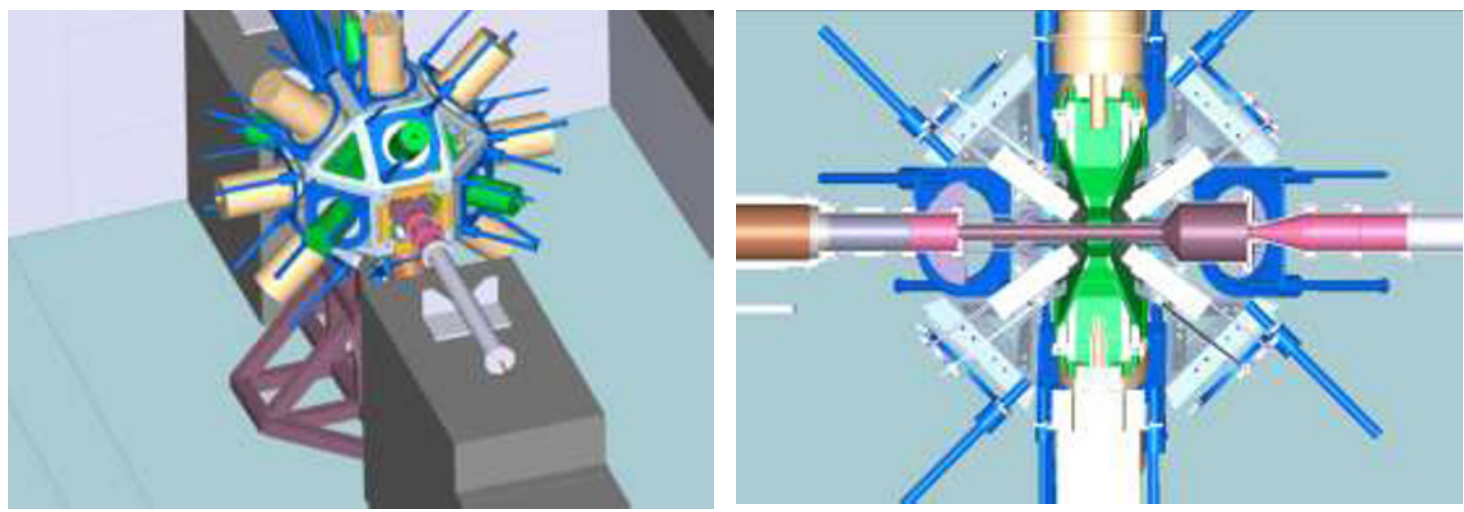

Figure 2. The two configurations of the EXILL campaign: Configuration 1, built from 8 EXOGAM large Clovers arranged in a ring perpendicular to the neutron beam, 6 coaxial detectors from GASP and two clovers from the ILL (left). Configuration 2, with 8 EXOGAM Clovers and $16 \mathrm{LaBr}_{3}$ for lifetime measurements (right).

The generation of angular momentum in the fission mechanism itself remains not understood. The angular correlations of $\gamma$-ray emitted by complementary fragments will give insights on how up to $20 \mathrm{~h}$ of angular momentum can be generated from an initial system with zero angular momentum. Of high interest to better understand fission is also the mass split and the prompt neutron multiplicity.

Finally, precise fission yields and prompt $\gamma$-ray spectra from ${ }^{235} \mathrm{U}(\mathrm{n}, \mathrm{f})$ and ${ }^{241} \mathrm{Pu}(\mathrm{n}, \mathrm{f})$ are crucial for calculating the heating in nuclear reactors and have thus strong impact on the design of the future reactors and fuel elements.

\subsection{The setup}

Two setups were envisaged (see figure 2). The first one consisted in 8 EXOGAM large clovers [3], 6 large coaxial detectors from GASP [4] and the 2 clovers from the ILL. In the second setup, the GASP and ILL detectors were replaced by $16 \mathrm{LaBr}_{3}$ crystals from the FATIMA collaboration [5]. The EXOGAM and GASP detectors were mounted with their anti-Compton shield. Both configurations were instrumented with a full digital triggerless data acquisition system based on 14 bit $100 \mathrm{MHz}$ CAEN digitizers. Details concerning the electronics and data acquisition systems can be found in [6].

The two setups were installed around the target point of the PF1B line of the ILL. The ILL reactor is the world's brightest continuous neutron source with an in-pile flux up to $1.5 \times 10^{15}$ $\mathrm{n} . \mathrm{s}^{-1} . \mathrm{cm}^{-2}$. Neutrons are guided first via the $\mathrm{H} 113$ ballistic neutron guide [7] toward the experimental area delivering a thermal-equivalent flux of $2 \times 10^{10} \mathrm{n} . \mathrm{s}^{-1} \cdot \mathrm{cm}^{-2}$ with a very large beam profile of $20 \times 12$ $\mathrm{cm}^{2}$. This beam section is reduced to a disk of roughly $1.2 \mathrm{~cm}$ diameter at the target position in order to 1) allow a compact arrangement of the $\gamma$-ray detectors around the target to maximize the detection efficiency, 2) perform precise spectroscopic studies like angular correlations and 3) avoid radiative capture reactions on the infrastructure items surrounding the detectors, which would cause a large background. The neutron beam collimation is realized by using a series of lithium and boron collimators mounted upstream the target. In order to produce around $10^{4}$ fissions per second in a 400 $\mu \mathrm{g}$ thick, $1 \mathrm{~cm}^{2},{ }^{235} \mathrm{U}$ target a flux of $10^{8} \mathrm{n} . \mathrm{s}^{-1} \cdot \mathrm{cm}^{-2}$ is required. Details of the collimation can be found in [8]. The campaign covered two reactor cycles (95 days) organized as follows: 21 (15) days for spectroscopy studies with ${ }^{235} \mathrm{U}\left({ }^{241} \mathrm{Pu}\right) ; 14$ (9) days for fast timing measurements using ${ }^{235} \mathrm{U}\left({ }^{241} \mathrm{Pu}\right)$ targets; during the remaining time $23(\mathrm{n}, \gamma)$ reactions were performed. 


\subsection{Experimental methods and performances}

In the absence of any fragment identification, it is crucial to acquire high-fold events ( 3 or larger) to possibly associate the observed $\gamma$-rays to a given fragment. This technique relies on the knowledge of the transitions deexciting the first excited states in the two fragments and the measurements of the evolution of the unknown $\gamma$-rays intensity gated by known transitions in a series of isotopes. ${ }^{235} \mathrm{U}$ and ${ }^{241} \mathrm{Pu}$ targets have been used leading for a given nucleus to two distinct complementary fragments. This will greatly help to confirm the assignment. The method relies therefore primarily on the efficient detection of high-fold data, which is achieved using a large number of efficient HPGe detectors. Such a technique is now well established and has been proven to work very well to analyze fission data [9, 10]. The total photopeak efficiency for the $16 \mathrm{Ge}$ detectors setup was about $6 \%$. The granularity is also crucial: even though $2 / 3$ of this efficiency is coming from the EXOGAM clover, the gain in the number of $\gamma-\gamma-\gamma$ coincidences obtained by adding the GASP and the ILL detectors is a factor of 4.2.

As an example we can compare the spectra obtained after setting two gates to select ${ }^{92} \mathrm{Rb}$ in ${ }^{248} \mathrm{Cm}$ fission data obtained with EUROGAM2 (10 days experiment) and our EXILL dataset. A gain of 30 in statistics is obtained. The second setup using the $\mathrm{LaBr}_{3}$ detectors was dedicated to lifetime measurements. In this configuration, the EXOGAM detectors are used to select the fragment of interest and the $\mathrm{LaBr}_{3}$ crystals give the lifetimes via the electronics fast timing technique using $\mathrm{LaBr}_{3}$ $\mathrm{LaBr}_{3}$ coincidences [11-13]. This method requires therefore $\mathrm{Ge}-\mathrm{LaBr}_{3}-\mathrm{LaBr}_{3}$ or $\mathrm{Ge}-\mathrm{Ge}-\mathrm{LaBr}_{3}-\mathrm{LaBr}_{3}$ coincidences. Recently, a novel method to analyze such data has been developed [14] and lead to the determination of lifetimes with an accuracy of 5 ps between $40 \mathrm{keV}$ and $6.7 \mathrm{MeV}$.

\subsection{Acnowledgments}

The EXILL campaign would not have been possible without the support of several services at the ILL and the LPSC.We are grateful to the EXOGAM collaboration for the loan of the detectors, to GANIL for assistance during installation and dismantling, and to the INFN Legnaro laboratory for the loan of the GASP detectors.

\section{References}

[1] M.A.C. Hotchkis et al., Phys. Rev. Lett. 64, 3123 (1990)

[2] JEFF-3.1.1 database, http://www.oecd-nea.org/dbdata/jeff/

[3] J.Simpson and the EXOGAM collaboration, Acta Physica Hungarica, New Series, Heavy Ion Physics 11, 159 (2000).

[4] D.Bazzacco, and the GASP Collaboration, Proc. Int. Conf. Nuclear Structure at High Angular Momentum, Ottawa, AECL-10613 p.376 (1992)

[5] http://nuclear.fis.ucm.es/fasttiming/

[6] P. Mutti et al., Proc. of the ANNIMA Conference, (2013)

[7] H. Abele et al., Nucl. Instr. and Meth. A 562407 (2006)

[8] W. Urban et al., JINST 8, 03014 (2013)

[9] M.A.C. Hotchkis et al., Nucl. Phys. A 530, 111 (1991)

[10] I. Ahmad and W.R. Phillips, Rep. Prog. Phys. 58, 1415 (1995)

[11] K. E. G. Löbner The electromagnetic interaction in nuclear spectroscopy, (ed. W. D. Hamilton, North-Holland New York 1975) 173

[12] H. Mach, R. L. Gill, M. Moszy’nski, Nucl. Instr. and Meth. A 28049 (1989)

[13] J.-M. Régis et al., Nucl. Instr. and Meth. A 62283 (2010)

[14] J.-M. Régis et al., Nucl. Instr. and Meth. A 68436 (2012) 